\title{
Pesquisa e atividades de extensão em fitoterapia desenvolvidas pela Rede FitoCerrado: uso racional de plantas medicinais e fitoterápicos por idosos em Uberlândia-MG
}

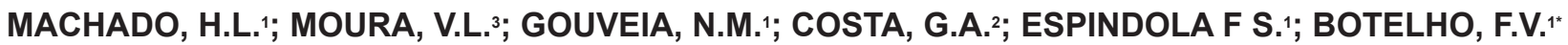 \\ 1. Universidade Federal de Uberlândia (UFU), Instituto de Genética e Bioquímica, Av. Pará 1720, Bloco 2E, \\ Umuarama, CEP 38400902, Uberlândia, MG, Brasil; 2Universidade Federal de Uberlândia (UFU), Faculdade

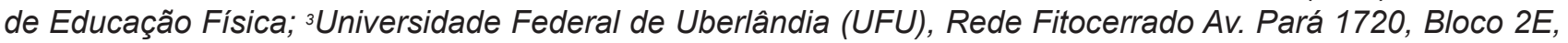 \\ Umuarama, CEP 38400902, Uberlândia, MG, Brasil
}

\begin{abstract}
RESUMO: Este estudo objetivou realizar atividades de extensão em fitoterapia, a partir de um levantamento de dados sobre as plantas medicinais, fitoterápicos e medicamentos convencionais utilizados por 292 idosos frequentadores de um programa de Atividades Físicas e Recreativas para a Terceira Idade (AFRID), na cidade de Uberlândia-MG, utilizando como instrumento de investigação, um questionário semiestruturado. Dentre os entrevistados verificamos que $88 \%$ utilizavam medicamentos prescritos, principalmente para o controle da hipertensão. O uso de plantas medicinais foi relatado por $76,7 \%$ dos idosos, sendo as mais citadas: Cymbopogon citratus, Mentha sp., Rosmarinus officinalis, Plectranthus barbatus, Ocimum gratissimum, e Matricaria chamomilla. Dezesseis $(5,5 \%)$ idosos utilizavam fitoterápicos, principalmente preparados a partir de extratos de Ginkgo biloba, Aesculus hippocastanum e Passiflora incarnata em associação com Crataegus oxyacantha e Salix alba. O uso concomitante de plantas medicinais e fitoterápicos com medicamentos convencionais foi relatado por $86,2 \%$ e $81,3 \%$ dos idosos, respectivamente. Após a análise dos dados percebemos a necessidade do desenvolvimento de ações educativas para informar e conscientizar os idosos sobre o uso da fitoterapia. Elaboramos uma caderneta e uma cartilha para promoção da difusão dessas informações e o aprimoramento do uso da fitoterapia entre os idosos e, dessa forma, alcançar os profissionais de saúde sobre os riscos e benefícios dessa terapêutica; contribuindo assim para o uso seguro e racional da fitoterapia.
\end{abstract}

Palavras-chave: Plantas medicinais, Fitoterápicos, Idosos, Interações medicamentosas, Atividades de extensão.

ABSTRACT: Research and extension activities in herbal medicine developed by Rede
FitoCerrado: rational use of medicinal plants by the elderly in Uberlândia-MG. This study
aimed to carry out extension activities in herbal medicine from a survey of data on medicinal
plants, herbal and conventional medicines used by 292 elderly people who attended a program of
physical activity called Physical and Recreational Activities for the Elderly, in Uberlândia-MG, using
a semi-structured questionnaire as means of investigation. Among the respondents $88 \%$ used
prescription drugs, primarily for control of hypertension. The use of medicinal plants was reported
by $76.7 \%$ of the elderly, being the most cited ones: Cymbopogon citratus, Mentha sp., Rosmarinus
officinalis, Plectranthus barbatus, Ocimum gratissimum. and Matricaria chamomilla. Sixteen
respondents (5,5\%) used herbal medicines, especially those prepared from extracts of Ginkgo
biloba, Aesculus hippocastanum, and Passiflora incarnata L. in association with Crataegus
oxyacantha L. and Salix alba. The concomitant use of medicinal plants and herbal medicines
with conventional drugs was reported by $86.2 \%$ and $81.3 \%$ of participants, respectively. After
analyzing the data, we detected the need to develop educational activities to inform and educate
seniors about the use of herbal medicine, encompassing the development a book and a primer
for initial dissemination of this information, improving herbal medicine use among the elderly.
This course of action would allow a greater knowledge of health professionals about the risks
and benefits of this therapy, thereby contributing to the safe and rational use of herbal medicine.

Keywords: Medicinal plants, Herbal Medicines, Elderly, Drug interactions, Extension activities

Recebido para publicação em 04/09/2013

Aceito para publicação em 24/03/2014

10.1590/1983-084X/13_072

Rev. Bras. PI. Med., Campinas, v.16, n.3, p.527-533, 2014. 


\section{INTRODUÇÃO}

O Brasil vivencia um processo de envelhecimento demográfico com particularidades que o destacam na escala mundial. De acordo com os dados do Instituto Brasileiro de Geografia e Estatística (IBGE), em dez anos, o número de idosos passou de 15,5 milhões (2001) para 23,5 milhões (2011) e o percentual de pessoas com 60 anos ou mais passou de $9,0 \%$ para $12,1 \%$ nesse período (IBGE, 2012).

Com envelhecimento aumenta-se a prevalência de doenças agudas ou crônicas, acompanhadas por uma maior demanda aos serviços de saúde e considerável aumento no consumo de medicamentos. Tais fatores predispõem a população geriátrica aos riscos da polifarmácia e a ocorrência de efeitos adversos e de possíveis interações medicamentosas (Secoli, 2010; Souza et al., 2008).

Apesar do avanço na medicina alopática, a Organização Mundial de Saúde (OMS) reconhece que grande parte da população dos países em desenvolvimento dependa da fitoterapia para sua atenção primária, tendo em vista que $80 \%$ desta população recorrem às práticas tradicionais nos seus cuidados básicos de saúde e $85 \%$ destes utiliza plantas medicinais (Brasil, 2006). Estudos mostram que terapias à base de plantas medicinais são amplamente utilizadas principalmente pelos idosos (Canter \& Ernst, 2004; Gama \& Silva, 2006; Loya et al., 2009; Marliére et al., 2008; Rosa et al., 2012).

Uma das preocupações com relação ao uso de plantas medicinais e fitoterápicos advém do fato de que muitos usuários têm a falsa ideia de que tais produtos são inócuos e que não apresentam potencial de toxicidade por serem "naturais" (Oliveira \& Gonçalves, 2006; Veiga Júnior et al., 2005). Com tal premissa, parte considerável dos usuários não revela aos prescritores sobre a utilização de produtos à base de plantas medicinais (Canter \& Ernst, 2004; Gold et al., 2001; Izzo, 2005; Pereira, 2008).

Os efeitos adversos decorrentes do uso de plantas podem ocorrer através das interações dos próprios constituintes das plantas medicinais/ fitoterápicos com outros medicamentos, ou ainda relacionados às características do paciente (idade, sexo, condições fisiológicas, entre outros). A identificação errônea das espécies vegetais, forma de preparo incorreta e o uso indiscriminado podem ser perigosos, levando a superdosagem, ineficácia terapêutica e efeitos indesejáveis, o que pode acarretar sérios danos ao usuário com o comprometimento da recuperação de sua saúde (Balbino \& Dias, 2010; WHO, 2004).

Considerando que os idosos são os principais usuários de plantas medicinais/fitoterápicos e que a maioria necessita de fazer uso diariamente de medicamentos convencionais, o objetivo do trabalho foi realizar um levantamento de dados sobre as principais plantas medicinais/fitoterápicos e medicamentos convencionais utilizados por idosos frequentadores de um programa de Atividades Físicas e Recreativas para a Terceira Idade (AFRID), na cidade de Uberlândia-MG, visando a realização de um evento sobre plantas medicinais/fitoterápicos e saúde e atividades de extensão como oficinas com o intuito de esclarecer as dúvidas mais frequentes quanto à identificação botânica e diferenciação de espécies vegetais, forma correta de preparo de plantas medicinais e potenciais interações entre plantas medicinais/fitoterápicos e fármacos. Além disso, elaboramos uma cartilha e uma caderneta para servir de ferramenta para a conscientização e esclarecimento aos usuários de plantas medicinais/ fitoterápicos e profissionais de saúde quanto ao uso correto e racional da fitoterapia.

As atividades foram desenvolvidas por integrantes da Rede FitoCerrado que é uma entidade sem fins lucrativos fundada em 2006 em UberlândiaMG. Tal entidade desenvolve ações na comunidade e tem contribuído para a valorização, resgate do conhecimento popular e da utilização das plantas medicinais, por meio de materiais informativos, palestras, oficinas, simpósios, encontros sobre o tema plantas medicinais e fitoterapia envolvendo acadêmicos, sociedade civil e pesquisadores.

\section{MATERIAL E MÉTODO}

A etapa inicial desse trabalho foi caracterizada pelo levantamento de dados sobre as principais plantas medicinais, fitoterápicos e medicamentos convencionais utilizados por 292 idosos que frequentam o grupo AFRID da Faculdade de Educação Física da Universidade Federal de Uberlândia-MG.

O programa AFRID tem como objetivo proporcionar atividades físicas em diferentes modalidades para pessoas que estejam na faixa etária de 50 anos ou mais com abordagem recreativa, bem como palestras e estudos de cunho informativo, visando à melhoria da qualidade de vida, o bem estar físico, social e emocional.

A amostra do estudo foi constituída por todos os idosos, com idade de 60 anos ou mais, que possuíam cadastro atualizado no relatório de integrantes do grupo AFRID e que aceitaram em participar do estudo. A escolha de tal grupo foi pelo fato de termos acesso aos telefones dos frequentadores e, dessa forma, podermos fazer o rastreamento inicial antes das entrevistas. Para a realização das entrevistas foram feitas visitas 
domiciliares, a partir do agendamento realizado através de ligações telefônicas aos idosos.

Utilizamos como instrumento de coleta de dados um questionário semiestruturado, preenchido durante a entrevista domiciliar. $O$ questionário continha perguntas referentes aos aspectos socioeconômicos, uso de plantas medicinais, fitoterápicos e uso de medicamentos convencionais. Após a avaliação e organização dos dados foram realizadas oficinas educativas sobre plantas medicinais e fitoterápicos com o intuito de esclarecer as dúvidas e difundir o conhecimento sobre o uso seguro e racional da fitoterapia e elaborada uma caderneta: "Caderneta do Idoso" e uma cartilha: "Uso de plantas medicinais e fitoterápicos: um manual do uso consciente".

A elaboração da caderneta foi dividida em itens e aborda dados referentes à identificação do idoso, problemas de saúde, medicamentos, fitoterápicos e plantas medicinais utilizadas, alergia ou intolerância a algum medicamento ou alimento, medicamentos já utilizados e que foram suspensos, registro do controle de pressão arterial, glicemia e peso e a sua agenda de consultas. A caderneta foi baseada na "Caderneta de saúde da pessoa idosa" elaborada pelo Ministério da Saúde (Brasil, 2008) que não aborda nenhum item em relação à fitoterapia.

A cartilha foi elaborada, pelos componentes do grupo de pesquisa. Em reuniões discutimos o conteúdo, a forma de abordar o conteúdo ao público idoso, tipo e tamanho de letras, figuras, desenhos etc. O objetivo de tal criação foi disponibilizar aos idosos e aos profissionais de saúde um material com os cuidados na utilização de fármacos convencionais, plantas medicinais e fitoterápicos e alertar sobre as possíveis interações medicamentosas entre plantas medicinais e fitoterápicos com outros fármacos, visando evitar complicações geradas a partir do uso incorreto e concomitante dos mesmos, uma vez que as plantas medicinais e os fitoterápicos apresentam efeitos curativos e colaterais.

Esta pesquisa foi realizada no período de outubro/2010 a fevereiro de 2011 e aprovada pelo Comitê de Ética em Pesquisa da Universidade Federal de Uberlândia - UFU sob o Parecer $n^{\circ}$ 195/11.

\section{RESULTADOS E DISCUSSÃO}

Realizamos entrevistas domiciliares com 292 idosos, sendo $256(87,7 \%)$ do sexo feminino e $36(12,3 \%)$ do sexo masculino. A faixa etária dos entrevistados foi de 60 a 88 anos, com uma média de idade de 69,8 $\pm 5,9$ anos. Verificou-se que 257 (88\%) utilizavam medicamentos prescritos, principalmente para o controle da hipertensão arterial. Esse dado é semelhante ao encontrado em um estudo realizado no Nordeste do Brasil, no qual $80,3 \%$ dos idosos da área central faziam uso de pelo menos um medicamento prescrito (Coelho Filho et al., 2004) e de outro estudo em Belo Horizonte onde a prevalência do uso de medicamentos foi de $90,1 \%$ (Ribeiro et al., 2008).

A utilização de fitoterápicos foi relatada por $16(5,5 \%)$ entrevistados, sendo que os mais utilizados foram aqueles preparados a partir de extratos de Ginkgo biloba L. $(n=6)$, Aesculus hippocastanum $\mathrm{L}$. $(\mathrm{n}=2)$ e Passiflora incarnata $\mathrm{L}$. em associação com Crataegus oxyacantha L. e Salix alba L. $(n=2)$. Em relação aos fitoterápicos mais utilizados os dados se assemelham ao encontrado em um estudo realizado em Belo Horizonte em que o Ginkgo biloba e Aesculus hippocastanum foram também os mais usados pelos idosos (Marliére et al., 2008). A maioria dos idosos desconhecia esse tipo de medicação e não conheciam a denominação "fitoterápicos".

Quanto à utilização de plantas medicinais, verificou-se que $76,7 \%$ (224) dos entrevistados reportaram o uso de alguma planta medicinal. Estudos têm demonstrado essa elevada utilização de plantas medicinais por idosos. Em um Centro de Saúde da cidade de São Paulo verificou-se que $68 \%$ dos idosos que utilizavam os serviços de saúde também faziam o uso de plantas medicinais (Gama \& Silva, 2006). Em um hospital geriátrico em Brasília houve uma prevalência de $83,9 \%$ de pacientes em uso da fitoterapia (Pereira, 2008).

Alguns fatores que contribuem para a elevada utilização de plantas medicinais por idosos são: o baixo custo, fácil obtenção, difícil acesso aos medicamentos nos serviços de saúde, poucos efeitos adversos quando comparados a medicamentos convencionais, tradição cultural e preferência pelo natural (Gama \& Silva, 2006).

Dentre as espécies vegetais utilizadas, as mais citadas pelos idosos foram Cymbopogon citratus (DC.) Stapf $(n=133)$, Mentha sp. $(n=103)$, Rosmarinus officinalis L. $(\mathrm{n}=58)$, Plectranthus barbatus Andrews ( $\mathrm{n}=54)$, Ocimum gratissimum $\mathrm{L}$. $(n=44)$ e Matricaria chamomilla L. $(n=34)$. Segundo os dados levantados o uso de plantas medicinais concomitante com medicamentos prescritos foi de $86,2 \%$. Os dados são semelhantes ao do estudo realizado por Rosa et al. (2012), no qual o uso simultâneo de plantas medicinais e medicamentos alopáticos foi referido por $93,1 \%$ dos entrevistados.

Quando os idosos foram indagados se informavam ao médico sobre o uso de plantas medicinais a maioria $60,7 \%$ (136) declarou não informar. O nível de desconhecimento do prescritor só aumenta os riscos do paciente, uma vez que o médico pode errar seu diagnóstico em função 
das muitas interações possíveis entre as plantas e os medicamentos convencionais (Veiga Junior et al. 2005). A automedicação com plantas é particularmente preocupante quando realizada em conjunto com outros medicamentos, podendo levar a efeitos sinérgicos e interações não esperadas pelo médico (Veiga Junior, 2008). Há também uma grande dificuldade em determinar a causa de interações entre um fármaco e uma planta medicinal, principalmente quando essas ocorrem em pacientes polimedicados, como os idosos (Colalto, 2010).

Outro problema está relacionado à dificuldade em se distinguir reações adversas de eventos relacionados à qualidade das plantas, adulteração, contaminação, preparação incorreta ou estocagem inadequada e/ou uso inapropriado e irracional (Silveira, 2007). Além disso, erros de identificação botânica e modos de preparo são bastante comuns. Nesta pesquisa, várias espécies, muitas vezes de gêneros e/ou famílias distintas, foram referenciadas por um mesmo nome popular, como por exemplo, "erva cidreira", nome vulgar que se refere tanto para Cymbopogon citratus (DC.) Stapf (Poaceae) quanto para Lippia alba (Mill) N.Br. (Verbenaceae) e Melissa officinalis L. (Labiateae).

Em um trabalho realizado por Feijó et al. (2012), também foram identificados problemas em relação ao uso das plantas, como a forma de preparo inadequada, a procedência e o armazenamento inadequado. Tais fatores podem comprometer a qualidade, eficácia e os benefícios da utilização das plantas à saúde.

Vários problemas contribuem para a ocorrência de interações entre plantas e medicamentos, bem como pela baixa difusão desta informação na sociedade. Entre estes fatores destacam-se: os problemas de identificação das espécies vegetais; a escassez de estudos clínicos sobre interações medicamentosas; a falta de incorporação das plantas nos programas de farmacovigilância; a falta de atenção por parte dos prescritores sobre o consumo destes produtos e a falta de treinamento dos profissionais de saúde de identificarem episódios de interações medicamentosas (Futuro et al. 2004).

Outra questão a se considerar é que, infelizmente, as informações técnicas e científicas geradas dentro das universidades nem sempre chegam à população. Por isso, cabe aos pesquisadores divulgarem as indicações terapêuticas, toxicidade, contraindicações e possíveis interações entre plantas e medicamentos e orientar à população quanto ao uso correto das plantas medicinais/fitoterápicos, evitando seu uso indiscriminado. É necessária uma interação cada vez maior da universidade com a comunidade para que a população seja informada dos benefícios e riscos do uso da fitoterapia (Britto et al. 2008).

Nossa ação extensionista, após analisarmos os dados, foi retornar à população entrevistada nossos resultados. Realizamos oficinas educativas com idosos com o intuito de orientá-los quanto às possíveis interações e os riscos que as plantas medicinais e os fitoterápicos podem oferecer, quando utilizados de forma indevida. Nas oficinas os idosos aprenderam a reconhecer, identificar e diferenciar espécies vegetais conhecidas pelo mesmo nome popular, tais como erva cidreira, citronela e boldo; aprenderam a preparar uma pomada cicatrizante de calêndula e arnica e um sachê repelente com citronela. Eles também foram informados sobre as possíveis interações medicamentosas entre plantas medicinais/fitoterápicos com fármacos convencionais. As oficinas foram realizadas, visando esclarecer as dúvidas mais frequentes quanto à identificação botânica e diferenciação de espécies vegetais, forma correta de preparo de plantas medicinais e potenciais interações entre plantas medicinais/fitoterápicos e fármacos.

Também realizamos um evento sobre plantas medicinais/fitoterápicos e saúde: "XII Encontro da Rede FitoCerrado: Saúde, Beleza e Nutrição na Terceira Idade". Este evento foi direcionado aos idosos do grupo AFRID, a comunidade em geral, estudantes e profissionais de saúde. O XII Encontro da Rede FitoCerrado contou com palestras educativas sobre plantas medicinais e fitoterápicos, com o intuito esclarecer dúvidas e difundir o conhecimento sobre o uso adequado e seguro de plantas medicinais. Também foram abordadas questões relacionadas a cuidados na saúde e nutrição na terceira idade. Foram oferecidos serviços de aferição de pressão arterial e glicemia, cortes de cabelo, orientações sobre alimentação saúde e estética, massagens, danças, auriculoterapia e práticas de Tai Chi Chuan aos participantes.

Como materiais de divulgação, distribuímos a caderneta e a cartilha que desenvolvemos (Figura 1). A caderneta constitui numa importante ferramenta para o monitoramento da saúde do idoso. Sua função primordial é prestar assistência baseada nas informações sobre as condições da saúde e vida do paciente. É um documento que o idoso deve carregar sempre consigo quando for a alguma unidade de saúde para que haja um acompanhamento dos profissionais de saúde sobre as plantas medicinais, fitoterápicos e os medicamentos convencionais utilizados pelos idosos. Com tal documento, fica fácil avaliar as possíveis interações medicamentosas e identificar possíveis erros e interações medicamentosas entre medicamentos convencionais.

A cartilha elaborada traz informações de 

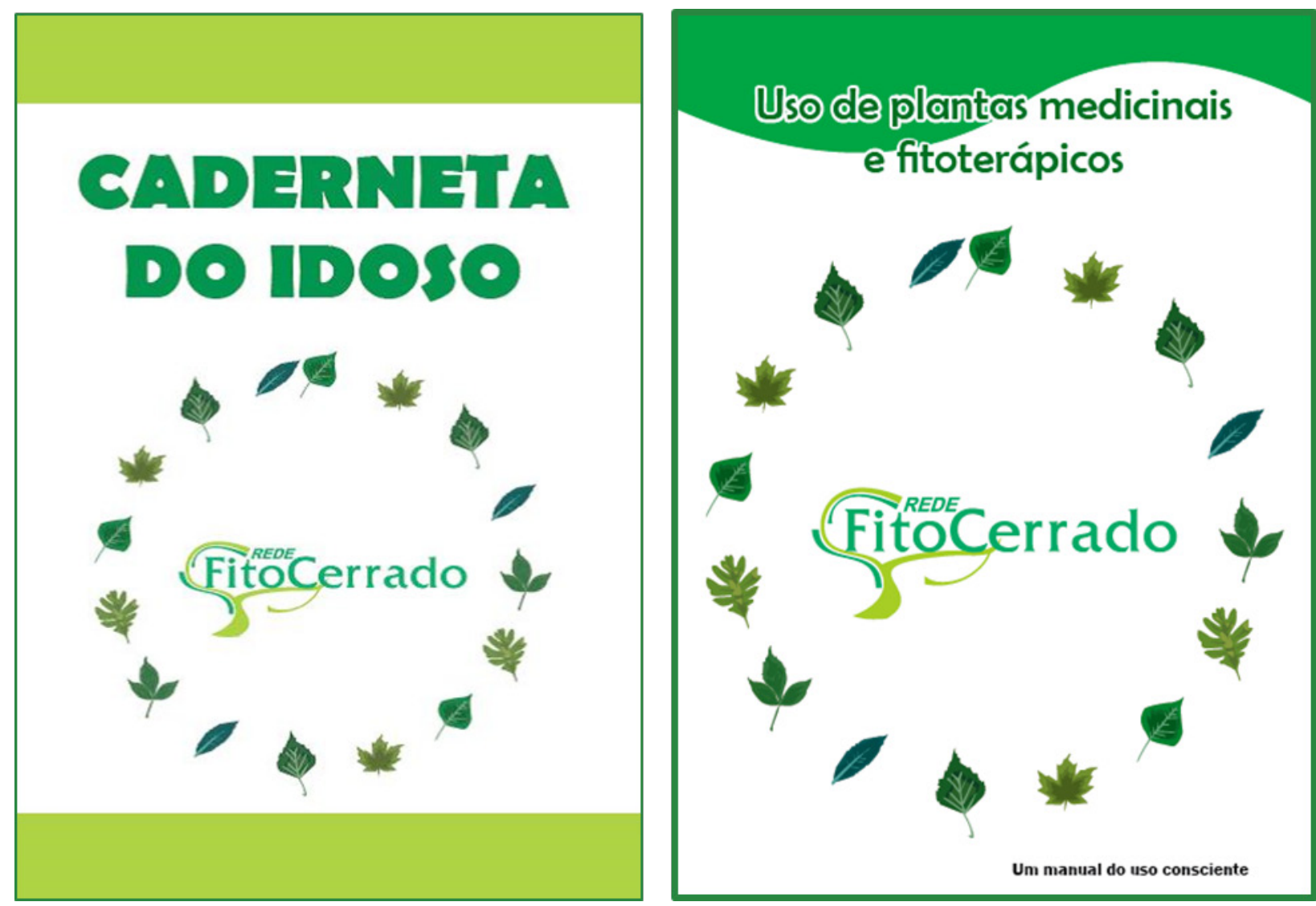

Figura 1. Capa da "Caderneta do Idoso" e da Cartilha "Uso de plantas medicinais e fitoterápicos".

indicações de usos, forma de preparo, cuidados na identificação botânica das plantas, contraindicações e possíveis interações medicamentosas entre fitoterápicos e plantas medicinais são mais utilizados e conhecidos pelos idosos: Cymbopogon citratus (DC.) Stapf., Cymbopogon nardus L., Melissa officinalis L., Lippia alba (Mill.) N.E.Br., Plectranthus barbatus Andrews, Vernonia condensata Baker, Mentha $x$ piperita L., Rosmarinus officinalis L., Zingiber officinale L., Matricaria recutita L., Ginkgo biloba L., Aesculus hippocastanum L., Glycine max L., e Passiflora incarnata L. O material desenvolvido é de fácil compreensão e vai servir de ferramenta para a conscientização e esclarecimento aos usuários de plantas medicinais/fitoterápicos e profissionais de saúde sobre a utilização correta e racional da fitoterapia.

O uso racional da fitoterapia é importante para a qualidade de vida dos idosos, mas para que isso ocorra é fundamental a orientação correta quanto ao uso da fitoterapia. Há necessidade de ações educativas que aprimorem a prescrição e o uso da fitoterapia entre idosos. Os usuários de plantas devem ser informados quanto à forma correta de preparo, posologia, dosagens adequadas, cuidados na identificação e armazenamento, para que se possa minimizar ou impedir a ocorrência de efeitos adversos ou de outros agravos à saúde decorrentes do uso inadequado de plantas medicinais.

\section{CONCLUSÃO}

Este trabalho permitiu o desenvolvimento das atividades extensionistas e o resgate do conhecimento popular sobre plantas medicinais entre idosos participantes do grupo AFRID na cidade de Uberlândia-MG. O estudo demonstra a importância da realização de atividades de extensão para aprimorar o uso da fitoterapia entre idosos e também com o intuito de que o conhecimento científico alcance a população para que haja maior divulgação e acesso a tais informações, interligando a universidade com a comunidade, promovendo a geração de conhecimentos entre ambos e proporcionando melhoria da saúde e qualidade de vida da população.

Pelas entrevistas e encontros realizados verificamos que os idosos possuem um grande conhecimento sobre plantas medicinais, mas notamos também que eles possuem muitas dúvidas e acreditam em mitos que precisam ser desmistificados. As plantas medicinais se utilizadas de forma adequada proporcionam inúmeros 
benefícios à saúde do idoso e, por isso, é importante disponibilizar as informações sobre plantas medicinais validadas cientificamente para que essas possam ser utilizadas pela população e também alertar sobre os cuidados na utilização de plantas medicinais. $\mathrm{O}$ uso da fitoterapia vinculado ao saber popular e a validação de seu uso é fundamental para garantir a segurança e eficácia de sua utilização como terapia complementar resgatando e potencializando o conhecimento tradicional.

A cartilha distribuída aos idosos foi o primeiro passo para que os mesmos possam ter o conhecimento mais científico. A caderneta visa propiciar um controle periódico das condições de saúde do idoso e de outros aspectos que possam interferir no seu bem-estar. Dessa forma, o preenchimento e o registro dos dados contidos na caderneta serão úteis para que os profissionais da saúde possam identificar riscos à saúde do idoso contribuindo para a melhoria da qualidade de vida do idoso com o uso racional de plantas medicinais e fitoterápicos associados ou não aos medicamentos convencionais.

\section{AGRADECIMENTOS \\ FitoCerrado \\ FAPEMIG, Idosos do grupo AFRID e Rede}

\section{REFERÊNCIA}

BALBINO, E.E.; DIAS, M. F. Farmacovigilância: um passo em direção ao uso racional de plantas medicinais e fitoterápicos. Revista Brasileira de Farmacognosia, v.20, n.6, p.992-1000, 2010.

BRASIL. Ministério da Saúde. Secretaria de Atenção à Saúde. Departamento de Atenção Básica. Política Nacional de Práticas Integrativas e Complementares no SUS - PNPIC-SUS. Brasília: Ministério da Saúde, 2006a. 92 p.

BRASIL. Ministério da Saúde. Caderneta de Saúde da Pessoa Idosa. Brasília: Editora do Ministério da Saúde, 2008. 24 p.

BRITTO, V.L. M.Q. et al. Plantas medicinais e fitoterápicos no contextoda academia, governo e organizações dasociedade civil: exemplo de iniciativas populares nomunicípio de Uberlândia-MG. Revista de Educação Popular, v.6, n.1, p.93-101, 2008.

CANTER, P.H.; ERNST, E. Herbal supplement use by persons aged over 50 years in Britain: frequently used herbs, concomitant use of herbs, nutritional supplements and prescription drugs, rate of informing doctors and potential for negative interactions. Drugs Aging, v.21, n.9, p.597-605, 2004

COELHO FILHO, J.M. et al. Perfil de utilização de medicamentos por idosos em área urbana do Nordeste do Brasil. Revista de Saúde Pública, v.38, n.4, p.557564, 2004.

COLALTO, C. Herbal interactions on absorption of drugs:
Mechanisms of action and clinical risk assessment. Pharmacological Research, v.62, n.3, p.207-227, 2010.

FEIJÓ, A.M. et al. Plantas medicinais utilizadas por idosos com diagnóstico de Diabetes mellitus no tratamento dos sintomas da doença.Revista Brasileira de Plantas Medicinais, v.14, n.1, p.50-56, 2012.

FUTURO, D.O. et al. Interações entre plantas e medicamentos. Informativo CEATRIM, Niterói, n. 3, p. 1-4, 2004.

GAMA, M.A.X.; SILVA, M.J.P. A utilização da fitoterapia por idosos de um Centro de Saúde em área central da cidade de São Paulo. Saúde Coletiva, v.11, n.3, p.79-84, 2006.

GOLD, J.L. et al. Herbal-drug therapy interactions: a focus on dementia. Current Opinion in Clinical Nutrition \& Metabolic Care, v.4, n.1, p.29-34, 2001.

IBGE. Síntese de Indicadores Sociais 2012.Uma análise das condições de vida da população brasileira. Rio de Janeiro: 2012. 293p. (Estudos \& Pesquisas - Informação demográfica e socioeconômica, 29). Disponível em: <http://www.ibge.gov.br>. Acesso em: 19 fev. 2013.

IZZO, A.A. Herb-drug interactions: an overview of the clinical evidence. Fundamental \& Clinical Pharmacology, v.19, n.1, p.1-16, 2005.

LOYA, A.M et al. Prevalence of polypharmacy, polyherbacy, nutritional supplement use and potential product interactions among older adults living on the United States-Mexico border: a descriptive, questionnaire based study. Drugs Aging, v.26, n.5, p.423-436, 2009.

MARLIÉRE, L.D.P. et al. Utilização de fitoterápicos por idosos: resultados de um inquérito domiciliar em Belo Horizonte (MG), Brasil. Revista Brasileira de Farmacognosia, v.18, suppl., p. 754-760, 2008.

OLIVEIRA, F.Q.; GONÇALVES, L.A. Conhecimento sobre plantas medicinais e fitoterápicos e potencial de toxicidade por usuários de Belo Horizonte, Minas Gerais. Revista Eletrônica de Farmácia, v.3, n.2, p. 36-41,2006.

PEREIRA, I.G.R. Prevalência do uso de fitoterapia em pacientes do programa em geriatria do Hospital Universitário de Brasília-HUB. 2008. 130p. Dissertação (Mestrado em Ciências da Saúde) - Faculdade de Ciências da Saúde, Universidade de Brasília, Brasília.

RIBEIRO, A.Q. et al. Inquérito sobre uso de medicamentos por idosos aposentados, Belo Horizonte, MG. Revista de Saúde Pública, v.42, n.4, p.724-732, 2008.

ROSA, R.L. et al. Investigação do uso de plantas medicinais no tratamento de indivíduos com diabetes melito na cidade de Herval D' Oeste - SC. Revista Brasileira de Plantas medicinais, v.14, n.1, p.50-56, 2012.

SILVEIRA, P.F. Perfil deutilização e monitorização de reações adversas a fitoterápicos do Programa Farmácia Viva em uma Unidade Básica de Saúde de Fortaleza. 2007. 143p. Dissertação (Mestrado em Ciências Farmacêuticas) - Faculdade de Farmácia, Odontologia e Enfermagem, Universidade Federal do Ceará, Fortaleza.

SECOLI, S.R. Polifarmácia: interações e reações adversas no uso de medicamentos por idosos. Revista Brasileira de Enfermagem, v.63, n.1, p.136-140, 2010.b

SOUZA, P.M. et al. Fármacos em idosos. In: BRASIL. 
Formulário terapêutico Nacional 2008: Rename 2006. Brasília: Ministério da Saúde, 2008. p. 26-29.

VEIGA JUNIOR, V.F. da. Estudo do consumo de plantas medicinais na região centro-norte do estado do Rio de Janeiro: aceitação pelos profissionais de saúde e modo de uso pela população. Revista Brasileira de
Farmacognosia, v.18, n.2, p. 308-313, 2008.

VEIGA JUNIOR, V.F. et al. Plantas medicinais: cura segura? Química Nova, v.28, n.3, p.519-528, 2005.

WHO.WHO guidelines on safety monitoring of herbal medicines in pharmacovigilance systems. Geneva: WHO, 2004. 68p. 